11

\title{
Возможности применения геометрической оптики для расчетов нано- и микроструктур в фотовольтаике
}

\author{
(C) E.В. Класс \\ Центральный научно-исследовательский институт химии и механики (ФГУП „ЦНИИХМ“), \\ 115487 Москва, Россия \\ e-mail: elenaklass@yandex.ru
}

Поступила в редакцию 21.05.2019 г.

В окончательной редакции 21.05.2019 г.

Принята к публикации 11.06.2019 г.

Оценки оптических характеристик, используемых в фотовольтаике нано- и микроструктур с помощью программ расчета переноса электромагнитного излучения показывают плохую сходимость результатов при больших затратах расчетного времени. Продемонстрирована возможность применения для этих целей приближения геометрической оптики. Для ряда представленных в научной литературе экспериментальных исследований по определению оптических характеристик нано- и микроструктур были проведены соответствующие расчеты с использованием трехмерной программы POKC-RG (метод Монте-Карло). Расчетные результаты показали удовлетворительное согласие с экспериментальными данными при учете в расчетах поверхностных шероховатостей, которые являются побочным результатом процессов травления при изготовлении структур. Анализ результатов также показал, что значительная частьизлучения поглощается за счет шероховатостей поверхности подложки структур. Указанная особенность должна приводить к неравномерности в распределении носителей заряда по высоте структуры и может служить одним из объяснений недостаточно высокой эффективности солнечных элементов с радиальной $p-n$-геометрией.

Ключевые слова: нано- и микроструктуры, фотовольтаика, оптические характеристики, геометрическая оптика, метод Монте-Карло, шероховатости.

DOI: $10.21883 /$ OS.2019.12.48700.199-19

\section{Введение}

Одним из направлений разработки конструкций солнечных батарей третьего поколения является использование структурированных элементов. Массивы из полупроводниковых нано- и микроструктур в виде цилиндров, конусов и т.п. фигур показывают существенное увеличение поглощающих свойств по сравнению с тонкопленочными образцами [1]. Отмеченная особенность была использована для разработки так называемых радиальных $p-n$-переходов, суть которых заключается в том, что внутри нанопроводов из кремния $n$-типа размещают ядро из кремния $p$-типа [2-4].

Повышение эффективности радиальной $p-n$-геометрии требует достаточно малых поперечных размеров фигур (порядка длины диффузии неосновных носителей). В то же время сокращение поперечных размеров имеет свой предел. При уменьшении диаметра отдельной фигуры увеличивается отношение площади ее поверхности к объему, что приводит к увеличению поверхностной рекомбинации. В итоге для разработанных конструкций параметры, характеризующие поперечные размеры отдельных фигур и расстояния между ними, имеют размеры от десятых долей до нескольких микрометров.

Расчет нано- и микроструктурных композиций обычно проводится с использованием программ переноса электромагнитного излучения [5-7]. Особенностью применения указанных программ для подобных объектов явля- ется плохая сходимость результатов и, как следствие, большие затраты расчетного времени. Следует отметить, что обычно нано- и микроструктурированные композиции получают с использованием различных модификаций метода химического травления, что в большинстве случаев приводит к шероховатости их поверхности $[8,9]$.

Приближение геометрической оптики при оценке оптических характеристик шероховатых поверхностей достаточно широко используется на практике ввиду простоты его реализации и небольших затрат расчетного времени по сравнению с методами на базе аппарата волновой оптики. Использование приближения геометрической оптики считается обоснованным в тех случаях, когда длина волны излучения меньше параметров, характеризующих структуру шероховатостей. Попытки расчетов реальных микроструктурных композиций на базе приближения геометрической оптики с использованием закона Ламберта для учета шероховатости поверхности проводов показали не слишком хорошие результаты [10]. В то же время сопоставления лучевого и волнового методов в применении к идеально гладким пирамидальным структурам показали удовлетворительное согласие для случаев, когда поперечный размер и период текстуры имеют размеры порядка длины волны [11].

В настоящей работе исследована возможность использования приближения геометрической оптики для определения оптических характеристик нано- и микроструктурированных композиций. Исследования проводились с 
помощью трехмерной программы метода Монте-Карло POKC-RG [12], которая позволяет учитывать шероховатость поверхностей. Корректность моделирования оценивалась на основе сопоставления результатов расчетов по программе POKC-RG с данными экспериментальных исследований по отражению оптического излучения от нано- и микроструктур различной формы, результаты которых достаточно подробно представлены в научной литературе.

\section{Особенности методики расчета}

Реализованный в POКC-RG подход, позволяющий оценить влияние шероховатых поверхностей на отражающие свойства объекта, имеет следующие особенности. Для описания трехмерного объекта в программе используется представление в виде зон, ограниченных поверхностями второго порядка. Для учета шероховатостей искомый объект математически описывается на двух уровнях: макроскопическом и микроскопическом. На макроуровне объект задается целиком в виде совокупности зон, имеющих гладкие поверхности. Геометрия микроуровня представляет собой подложку конечных размеров, на которой задаются неоднородности в виде тел вращения. Это могут быть эллипсоиды, конусы, параболоиды и т. П. При моделировании траектории светового кванта переход от макро- к микрогеометрии производится в случае, когда частица попадает на поверхность макрообъекта, которая помечена как шероховатая. Далее прослеживаются отражения частицы от неоднородностей микрогеометрии до выхода за ее пределы. После этого совершается обратный переход к макрогеометрии, и моделирование траектории кванта продолжается. В актах взаимодействия частицы с неоднородностями микроуровня может разыгрываться зеркальное или диффузное отражение, а вес отраженной частицы вычисляется по соответствующим формулам.

Положительным моментом использования приближения геометрической оптики является возможность при задании размеров расчетной схемы микрогеометрии пользоваться законом подобия. А именно результаты расчетов, полученные в трехмерной геометрии с линейными размерами $X \times Y \times Z$, можно распространять на геометрию с размерами $a(X \times Y \times Z)$, где $a$ коэффициент пропорциональности.

В ряде рассмотренных ниже экспериментов изготовленные наноструктуры содержали материалы, которые являются прозрачными или полупрозрачными для оптического излучения. В этой связи методический аппарат программы POKC-RG, изложенный в [12] и изначально ориентированный на расчет отраженного излучения от непрозрачных тел, был дополнен алгоритмами для расчета преломления излучения и его поглощения в материалах.

Поглощение оценивается по экспоненте закона Бугера-Ламберта, в которой показатель поглощения $\alpha$ вы- числяетсяв соответствии с выражением $\alpha=4 \pi k / \lambda$, где $k$ - коэффициент поглощения, $\lambda-$ длина волны. При моделировании преломленного излучения микрогеометрия используется только для определения направлений траекторий, преломленных на шероховатой границе поверхности структуры. А именно после перехода в микрогеометрию, моделирующую шероховатости искомой поверхности, новое направление траектории вычисляется по стандартным формулам закона Снеллиуса. Далее осуществляется возврат в основную геометрию, где и продолжается моделирование преломленной траектории.

Известно, что приближение геометрической оптики считается корректным в тех случаях, когда длина волны излучения меньше параметров, характеризующих структуру шероховатостей. В случае случайно шероховатой поверхности к таковым принято относить [13] корреляционную длину $\tau$ и среднеквадратичное отклонение (дисперсию) высот шероховатостей относительно среднего уровня $\sigma$. В работах Танга $[14,15]$ было показано, что приближение геометрической оптики достаточно хорошо согласуется с точными решениями волновых методик и экспериментальными данными и при менее строгих соотношениях между длиной волны и параметрами неоднородностей. А именно приближение геометрической оптики показывает корректные результаты в тех случаях, когда выполняются следующие соотношения: $\sigma \cos \left(\theta_{0}\right) / \lambda>0.17$ и $\sigma / \tau<2$, где $\theta_{0}-$ угол падения излучения относительно нормали к гладкой поверхности. Указанные выводы позволяют рассчитывать на адекватность результатов применения приближения геометрической оптики в нано- и микроструктурах.

Как указывалось выше, влияние шероховатостей в программе POKC-RG оценивается на основе микрогеометрий, состоящих из тел, поверхность которых описывается уравнениями поверхностей второго порядка. Указанное представление отличается от традиционного подхода приближения геометрической оптики, в котором шероховатости описываются микрогранями [16,17]. Корректность используемого нами описания подтверждает следующее. С одной стороны, из фотографий микроструктур видно, что шероховатости имеют округлые формы. С другой стороны, в достаточно давней работе 1975 г. [18] было показано, что выбор направления нормалей к микрограням из распределения, опирающегося на представлении о форме шероховатостей в виде эллипсоидов вращения, дает лучшее согласие с экспериментально полученным распределением для случайно шероховатой поверхности в сравнении с подходами, опирающимися на распределение Гаусса. А в работе [19] было показано, что функция распределения микронормалей, использующая эллипсоидальную форму шероховатостей, для некоторых случаев в наилучшей степени описывает характеристики преломленного излучения полупрозрачной случайно шероховатой поверхности. Следует также отметить, что поверхности с принудительной шероховатостью, полученные, например, в результате 
лазерной абляции, часто имеют конусообразную форму неоднородностей [20].

Степень шероховатости структур, описываемых уравнениями поверхностей второго порядка, достаточно сложно параметризировать. Иногда для этой цели используется так называемое аспектное отношение, которое определяется отношением глубины впадины между фигурами к расстоянию между ними. Однако для однозначной характеристики отражающей способности шероховатой поверхности указанная величина не слишком корректна, поскольку число многократных отражений от поверхности зависит от формы фигур, образующих шероховатости.

Исследования, проведенные в настоящей работе, показали, что поверхность, образованная эллипсоидами, практически всегда характеризуется однократным отражением, в то же время для поверхности из конусов, напротив, характерным является большое число взаимных отражений. В частности, например, для эллиптических шероховатостей с аспектным отношением $t=2$ при нормальном падении световых квантов отраженное излучение формируется практически только за счет первого отражения. А для конусообразной шероховатости доля излучения, сформированного за счет первого отражения, в этом случае составляет лишь $30 \%$. С увеличением высоты шероховатостей за счет эффектов взаимного экранирования доля первых отражений уменьшается и для конусов, и для эллипсоидов. Но для шероховатостей из эллипсоидов это уменьшение не так значительно, как для шероховатостей из конусов. В последнем случае, начиная с некоторой высоты, число первых отражений для малых углов падения становится близким к нулю.

В наших исследованиях при составлении микрогеометрий для учета различий в отражающей способности структур были использованы оба вида фигур: и эллипсоиды, и конусы.

\section{Сравнения с экспериментальными данными}

Первая из использованных работ - [21], в которой измерялись поглощающие характеристики наноструктур, сформированных на основе пленки из гидрогенизированного аморфного кремния. Рассмотренные наноструктуры представляли собой нанопроволоки и наноконусы со скругленными вершинами, полученные с использованием масок из кремнезема травлением с использованием хлора. Параметры структур, измеренные с помощью электронного микроскопа, имели следующие величины: расстояния между вершинами составили около $500 \mathrm{~nm}$, толщина нанопроволоки, а также диаметр основания наноконусов - примерно $300 \mathrm{~nm}$, высота фигур порядка $600 \mathrm{~nm}$. Поглощающую способность структур в [21] оценивали с помощью интегрирующей сферы, которая собирала все отраженное и проходящее излучение от образца, помещенного в центре сферы. Искомое поглощение авторы [21] получали вычитанием указанных компонентов измеренного излучения из падающего потока квантов.

В расчетных исследованиях макрогеометрия задачи была задана по аналогии с геометрией экспериментов по измерению коэффициентов отражения с помощью интегрирующей сферы. Искомый образец моделировался в виде пластины, толщина которой была существенно меньше ее поперечных размеров. Пластина окружалась сферой, с помощью которой регистрировались отраженные кванты. Фигуры, моделирующие наноструктуры, размещались на одной из торцевых поверхностей пластины с плотностью заполнения материалом структуры, соответствующей плотности заполнения в эксперименте. Оптические константы для расчета брались из [22]. В расчетах исследовалась зависимость поглощения от угла падения излучения с длиной волны $488 \mathrm{~nm}$. Для указанной длины волны аморфный гидрогенизированный кремний является непрозрачным, и для расчетного определения его поглощающих свойств достаточно определить коэффициент отражения.

На рис. 1, $a$ показаны результаты экспериментальных измерений из [21] для поглощающей способности образцов с наноцилиндрами в зависимости от угла падения излучения в сравнении с модельными расчетами по POKC-RG, в которых все поверхности структур полагались идеально гладкими с зеркальным или с диффузным отражением. Видно, что в целом расчет с идеально гладкими поверхностями слабо согласуется с экспериментальными данными. Но можно отметить, что при малых углах падения оценка с использованием закона отражения Ламберта ближе к экспериментальным значениям, чем оценка на основе законов зеркального отражения. В то же время общий характер зависимости зеркально отражающих поверхностей для углов падения, больших $30^{\circ}$, существенно ближе к экспериментальной зависимости, чем для диффузно отражающих поверхностей.

Изменения в поглощающей способности структуры, связанные с учетом шероховатости их поверхности, демонстрирует рис. $1, b$. Анализ фотографий наноструктур, представленных в [21], показал, что стенки структур достаточно гладкие, в то время как на подложке видны неоднородности. Поэтому было рассмотрено два случая: когда шероховатость имеет только поверхность подложки структуры, а также когда все поверхности являются шероховатыми. На рисунке представлены расчетные данные для шероховатостей эллипсоидального типа. Там же показаны и результаты расчета методом матрицы рассеяния [6], полученные авторами [21]. Видно, что результаты наших расчетов показывают удовлетворительное согласие с экспериментом, особенно для случая гладких стенок цилиндров и шероховатой подложки. Варьирование вида шероховатости показало, что конусообразные шероховатости способствуют увеличению поглощения в наноструктуре при малых углах падения. 

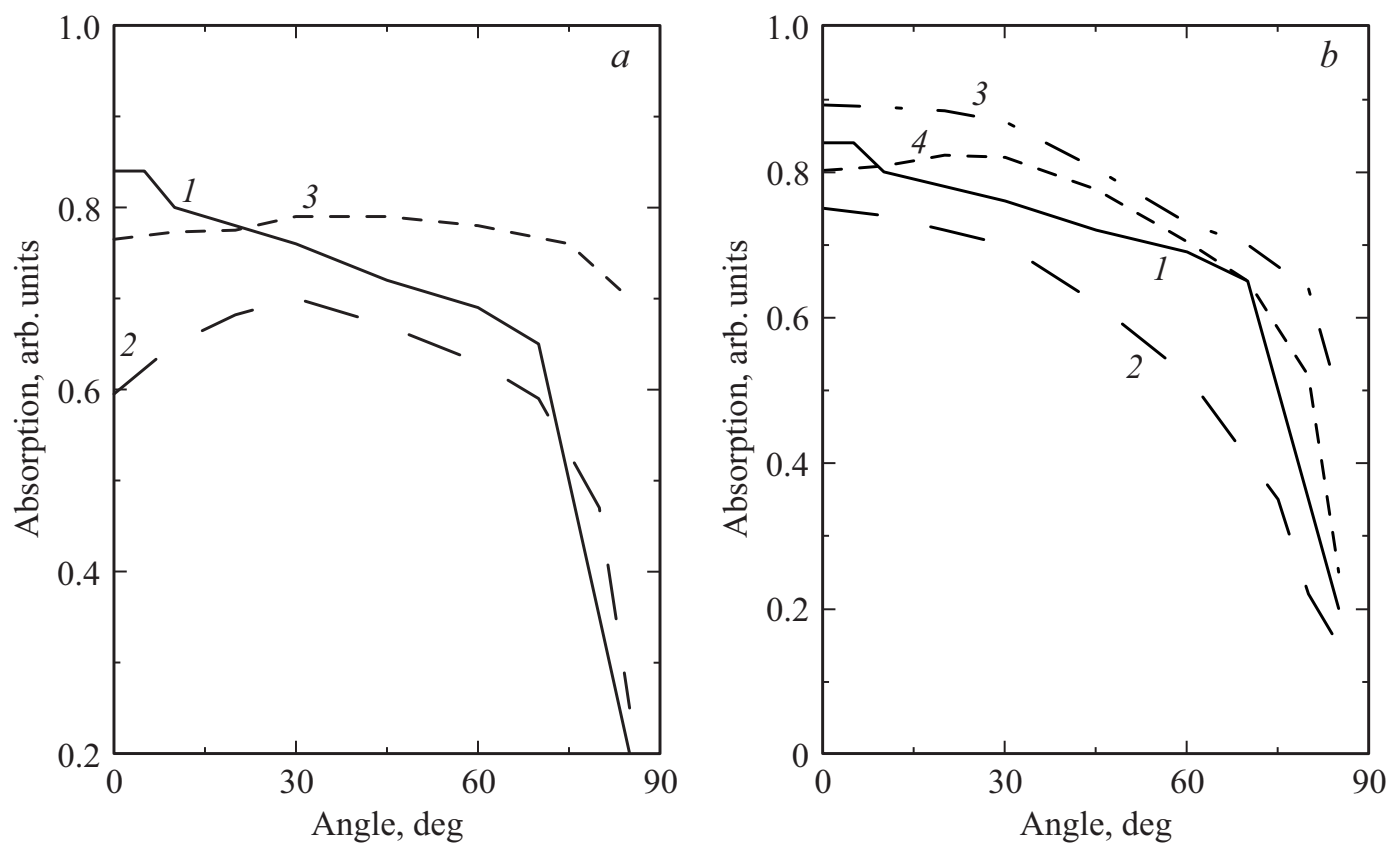

Рис. 1. Зависимость поглощения от угла падения излучения для структуры из наноцилиндров. (a) $1-$ эксперимент [21], 2,3 - расчет (POKC-RG) для структуры с гладкими стенками с зеркальным и диффузным отражением соответственно. $(b) 1-$ эксперимент [21], 2 - расчет [21], 3,4 - расчет (POKC-RG) с учетом шероховатости эллипсоидального типа на всей поверхности структуры и только на подложке соответственно.

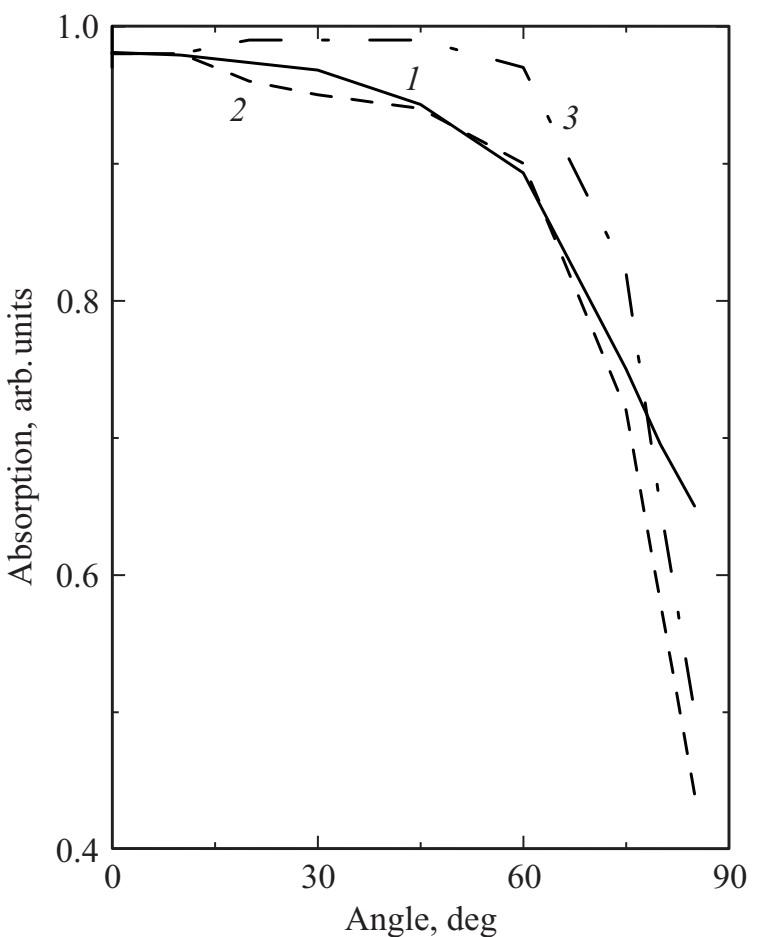

Pис. 2. Зависимость поглощения от угла падения для структуры из наноконусов. 1,3 - эксперимент и расчет [21] соответственно, 2 - расчет (РОКС-RG) с учетом шероховатостей.

Для углов падения, больших $45^{\circ}$, кривые поглощения для шероховатостей различных типов близки.
Сопоставления результатов расчетов по программе POKC-RG с экспериментальными данными [21] для структуры, образованной наноконусами, представлены на рис. 2. Шероховатости моделировались конусами с аспектным отношением, примерно равным 2. Видно достаточно хорошее согласие данных расчета по РОКС$\mathrm{RG}$ с экспериментальными данными [21] вплоть до $75^{\circ}$ относительно нормали к поверхности. Использование микрогеометрии с фигурами эллипсоидального типа уменьшает величину поглощения для нормального падения излучения с 0.97 до 0.9. При увеличении угла падения различие уменьшается, и при углах падения, больших $60^{\circ}$, поглощение практически не зависит от типа шероховатостей.

Следует отметить, что в обоих рассмотренных случаях идеально гладкие поверхности фигур и подложки при малых углах падения поглощают существенно меньше, чем структуры с шероховатыми поверхностями.

Следующая анализируемая работа [23] была посвящена исследованию зависимости процессов поверхностной рекомбинации для солнечных батарей, использующих кремниевые цилиндрические микроструктуры, размещенные на тонкой кремниевой мембране, от линейных размеров входящих в них фигур. В указанной работе были измерены спектральные зависимости поглощения для структур с микроцилиндрами различных диаметров: $0.54,0.74,0.94$ и $2 \mu \mathrm{m}$. Высота цилиндров для всех рассмотренных вариантов структур не менялась и составляла $10 \mu \mathrm{m}$, также как и не менялось и отношение высоты структур к периоду конфигурации. Толщина 


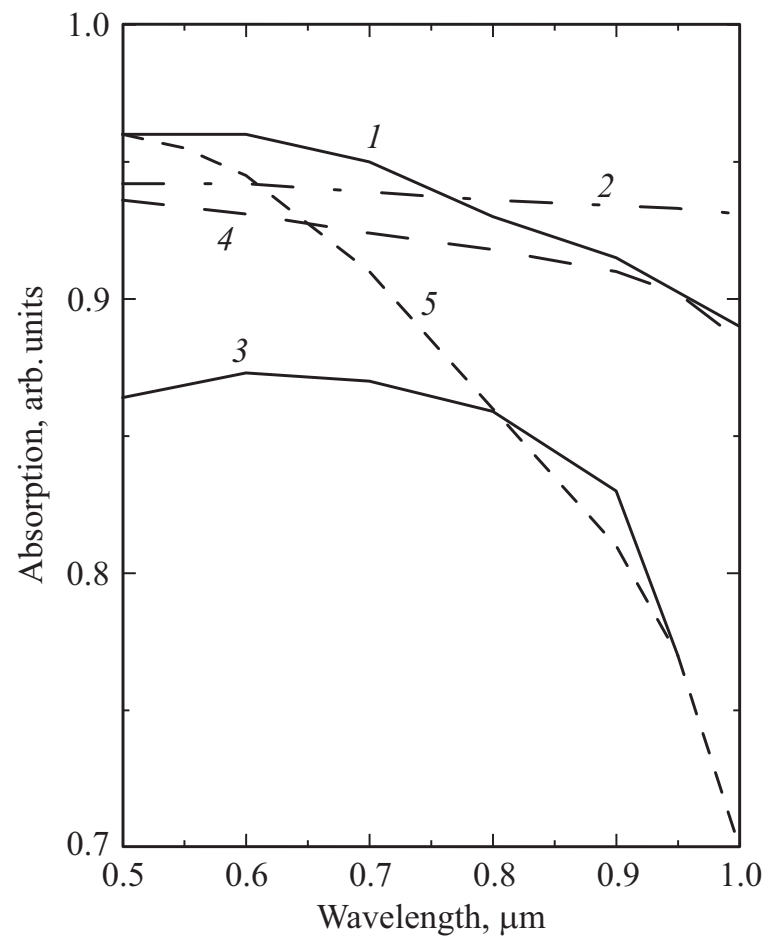

Рис. 3. Спектральная зависимость поглощения в цилиндрической микроструктуре из кремния: 1 - эксперимент [23], расчет (РОКC-RG): 2,3 - шероховатости на стенках и подложке (с АОП и без АОП соответственно), 4 - только на подложке (с АОП), 5 - поглощение в плоском слое кремния толщиной $50 \mu \mathrm{m}$, покрытого АОП [23].

мембраны, на которой размещались цилиндры, составила $40 \mu \mathrm{m}$. Поверхность изготовленных микроструктур была покрыта антиотражающим покрытием (АОП) из нитрида кремния. Экспериментальные измерения проводились в диапазоне длин волн $0.5-1.0 \mu \mathrm{m}$.

Расчет поглощения светового излучения по программе POKC-RG для структур из [23] проводили с использованием алгоритмов, учитывающих пропускание оптического излучения в материалы структуры. Для учета АОП коэффициент преломления материала структуры задавался для материала АОП, а коэффициент поглощения - для кремния. На рис. 3 показаны результаты расчетов для структуры из цилиндров диаметром $2 \mu \mathrm{m}$ в сравнении с результатами экспериментов [23]. Там же для наглядности представлены усредненные экспериментальные данные по поглощению излучения в неструктурированной мембране толщиной $50 \mu \mathrm{m}$, покрытой АОП. Приведенные расчетные данные получены с учетом шероховатостей эллипсоидального типа только на подложке (кривая 4), а также на всех поверхностях структуры (кривые 2 и 3 ). В последнем случае расчет проводился без учета АОП. Видно удовлетворительное согласие расчетных и экспериментальных данных. Для шероховатостей конусообразного типа результаты практически не зависят от длины волны, поглощение примерно равно 0.97.
Экспериментальные данные для фигур меньших диаметров мало различаются между собой. Так, максимальное различие для цилиндров диаметром 0.74 и $2 \mu \mathrm{m}$ в [23] составляет примерно 3\%. Данные наших расчетов для фигур меньших диаметров также практически не отличаются от приведенных.

В реальной практике параметры и конфигурации шероховатостей экспериментально полученных нано- и микроструктур обычно неизвестны. В рассматриваемом случае расчеты проводились для различных конфигураций использованных микрогеометрий (конусы, эллипсоиды). Исследования по оценке влияния аспектного отношения неоднородностей на величину коэффициента поглощения показали, что для шероховатостей в виде конусов при увеличении аспектного отношения $t$ в 2 раза коэффициент поглощения увеличивается от значения 0.97 до значения 0.98. Для эллипсоидальных шероховатостей указанное влияние еще менее значимо.

В заключение рассмотрим работу [24], которая посвящена экспериментальному исследованию отражения от кремниевых наноструктур с целью выбора рационального дизайна для эффективного поглощения светового излучения. В указанной работе было получено несколько типов наноструктур (цилиндры, конусы, цилиндр, сочлененный с конусом, и цилиндр, сочлененный с полусферой). Диаметр фигур составлял $0.8 \mu \mathrm{m}$, высота $-2 \mu \mathrm{m}$, период размещения фигур $-1.27 \mu \mathrm{m}$. Отражение измерялось в диапазоне длин волн $0.4-1.0 \mu \mathrm{m}$.

На рис. 4 представлено сопоставление результатов эксперимента по определению коэффициента отражения наноструктуры из цилиндров с данными расчетов по программе POKC-RG в зависимости от длины волны излучения. На этом же рисунке для наглядности представлена соответствующая зависимость для бесконечно толстого плоского слоя кремния. В расчетах предполагалось, что поверхности цилиндров гладкие, а поверхность подложки имеет конусообразный или эллипсоидальный тип шероховатости. Видно, что конусообразные шероховатости обеспечивают удовлетворительное согласие с экспериментальными данными. Результаты расчетов в предположении шероховатостей эллипсоидального типа выше экспериментальных данных примерно в два раза. Но, несмотря на указанное различие, видно, что ход спектральной зависимости и в этом случае повторяет экспериментальную. Учет шероховатости на всей поверхности структуры, как показали расчеты, меняет величину отражения от структуры примерно на $3-4 \%$. Соответствующие кривые на рисунке не показаны во избежание загромождения поля рисунка.

Расчетные данные, полученные в [24] с использованием волновых методик, демонстрируют в рассмотренном диапазоне длин волн осциллирующую кривую с размахом колебаний порядка двух раз, а ее усредненное значение лежит в основном выше экспериментальных данных. Указанная кривая также во избежание загромождения не представлена на рисунке. 


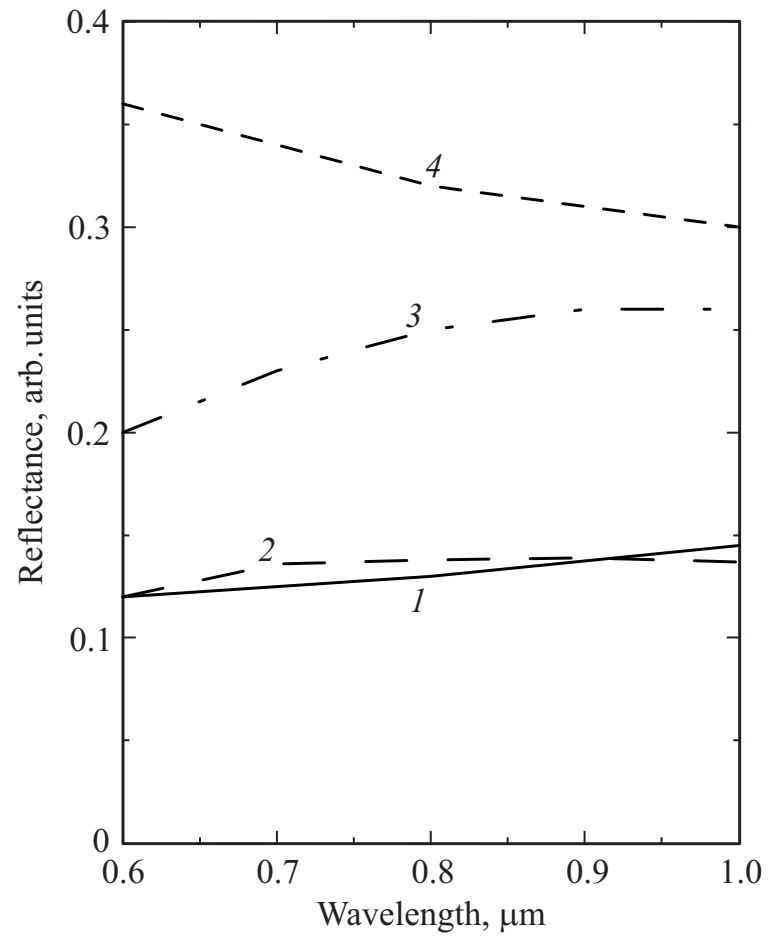

Pис. 4. Спектральная зависимость отражения для цилиндрической наноструктуры из кремния. 1 - эксперимент [24], 2,3 - расчет (POKC-RG) структуры с учетом шероховатостей на подложке конусообразного и эллиптического типов соответственно, 4 - отражение от плоской пластины [24].

Расчеты для наноструктур, имеющих форму заточенного карандаша (сочленение цилиндра и конуса), также показали хорошее согласие с экспериментальными данными. В эксперименте для указанной наноструктуры был получен коэффициент отражения примерно равный 0.03 , а в наших расчетах в предположении гладкости поверхности „карандашей“ и шероховатости подложки конусного типа было получено значение порядка 0.02 .

В работе [24] также представлены данные для наноструктуры примерно вдвое меньших размеров: диаметр $-0.38 \mu \mathrm{m}$, период $-0.6 \mu \mathrm{m}$ с тем же коэффициентом заполнения материалом. Анализ приведенных экспериментальных значений спектральной зависимости коэффициентов отражения для двух структур с различающимися по абсолютной величине параметрами и одинаковым коэффициентом заполнения материалом показывает их малозначимое различие. Это может свидетельствовать о том, что при формировании отраженного излучения от наноструктур подобных размеров доминирует чисто геометрическое ослабление.

В настоящее время эффективность солнечных элементов с радиальной $p-n$-геометрией не соответствует достигнутому высокому уровню поглощения светового излучения в указанных элементах. При увеличении поглощающей способности в разы рост тока короткого замыкания составляет не более $30 \%$ [25,26]. Анализ представленных в настоящей работе результатов расчетных исследований показал, что на поглощение излучения в нано- и микроструктурах в значительно большей степени влияет шероховатость поверхности подложки, чем шероховатость поверхности самих фигур. Это означает, что носители заряда образуются неравномерно по высоте структуры, основная их часть будет сосредоточена вблизи подложки (кроме, естественно, верхней торцевой части структур). Указанная неравномерность может служить одним из объяснений недостаточного уровня эффективности солнечных элементов с радиальной $p-n$-геометрией.

\section{Заключение}

Сопоставления с данными экспериментальных исследований, представленные в работе, свидетельствуют об адекватности результатов, получаемых с использованием предложенного способа расчета оптических характеристик нано- и микроструктур, учитывающего шероховатость их поверхностей. Временные затраты разработанной методики, использующей приближение геометрической оптики, несопоставимы с затратами на расчет с помощью программ переноса электромагнитного излучения: это единицы минут в сравнении с десятками часов, что позволяет рекомендовать его для определения оптимальных вариантов конструкций нанои микроструктур. Показано, что значительный вклад в поглощение оптического излучения в исследованных структурах вносит шероховатость поверхности подложки. Это может приводить к пространственной неравномерности носителей заряда по высоте структуры и служить одной из причин недостаточной эффективности солнечных элементов с радиальной $p-n$-геометрией.

\section{Финансирование работы}

Работа выполнена в инициативном порядке.

\section{Конфликт интересов}

Автор заявляет, что конфликта интересов нет.

\section{Список литературы}

[1] Tsakalakos L., Balch J., Fronheiser J., Shih M., LaBoeuf S., Pietrzykowski M., Codella P., Korevaar B., Sulima O., Rand J., Davuluru A., Ropol U. // J. Nanophoton. 2007. N 1. P. 013552.

[2] Rui Yu, Qingfeng Lin, Siu-Fung Leung, Zhiyong Fan // Nano Energy. 2012. N 1. P. 57.

[3] Garnett E., Yang P. // J. Am. Chem. Soc. 2008. V. 130. P. 9224.

[4] Kayes B., Atwater H., Lewis N. // J. Appl. Phys. 2005. V. 7. P. 114302.

[5] Oskooi A.F., Roundy D., Ibanescu M., Bermel P., Joannopoulos J.D., Johnson S.G. // Comput. Phys. Commun. 2010. V. 181. P. 687. 
[6] Tikhodeev S.G., Yablonskii A.L., Muljarov E.A., Gippius N.A., Ishihara T. // Phys. Rev. B. 2002. V. 66. N 4. P. 045102.

[7] Taflove A., Hagness S.C. Computational Electrodynamics: The Finite-Difference Time-Domain Method. Boston, MA: Artech House, 2005.

[8] Lin H., Cheung H.-Y., Xiu F., Wang F., Yip S., Han N., Hung T., Zhou J., Ho J.C., Wong C.-Y., Mater K. // J. Chem. A. 2013. V. 1. P. 9942.

[9] Putnam M.C., Boettcher S.W., Kelzenberg M.D., TurnerEvans D.B., Spurgeon J.M., Warren E.L., Briggs R.M., Lewis N.S., Atwater H.A. // Energy Environ. Sci. 2010. V. 3. P. 1037.

[10] Kosten E.D., Warren E.L., Atwater H.A. // Optics Express. 2011. V. 19. N 4. P. 3316.

[11] Eyderman S., John S., Deinega A. // J. Appl. Phys. 2013. V. 113. P. 154315.

[12] Класс Е.В., Шаховский В.В., Бадюк К.В., Ульянов С.А. // Оптический журнал. 2014. Т. 82. № 2. С. 3.

[13] Басс Ф.Г., Фукс И.М. Рассеяние волн на статистически неровной поверхности. М.: Наука, 1972. 424 с.

[14] Tang K., Dimenna R.A., Buckius R.O. // Int. J. Heat Mass Transfer. 1997. V. 40. P. 49.

[15] Tang K., Buckius R.O. // Int. J. Heat Mass Transfer. 1998. V. 41. P. 2037.

[16] Torrance K.E., Sparrow E.M. // JOSA. 1967. V. 57. N 9. P. 1105-1114.

[17] Cook R.L., Torrance K.E. // Computer Graphics. August 1981. V. 15. N 3. P. 307-316.

[18] Trowbridge T.S., Reitz K.P. // J. Opt. Soc. Am. 1975. V. 65. P. 531-536.

[19] Bruce W., Marschner S.R., Li H., Torrance K.E. // Eurographics Symposium on Rendering. 2007. P. 195-206. http://www.cs.cornell.edu/srm/publications/EGSR07-btdf.html

[20] Воронов В.В., Долгаев С.И., Лаврищев С.В., Лялин А.А., Симакин А.В., Шафбеев Г.А. // Квант. электрон. 2000. Т. 30. № 8. C. $710-714$.

[21] Zhu J., Yu Z., Burkhard G.F., Ching-Mei Hsu, Connor S.T., Xu Y., Wang Q., McGehee M., Fan S., Cui Y. // J. Nano Lett. 2009. V. 9. N 1. P. 279.

[22] Palik E.D., Ghosh G. Handbook of Optical Constants of Solids. V. 3. Academic, 1998. 3187 p.

[23] Shin J.Ch., Chanda D., Chern W., Yu K.Ju., Rogers J.A., Li X. // J. Photovoltaics. 2012. V. 2. N 2. P. 129.

[24] Lin H., Xiu F., Fang M., Yip S.P., Cheung Ho-Yu., Wang F., Han N., Chan K.S., Wong Ch-Yu., Ho J.C. // ACS Nano. 2014. V. 8. P. 3752. www.acsnano.org

[25] Adachi M.M., Anantram M.P., Karim K.S. // Scientific Reports. 2013. V. 3. Article number 1546. www.nature.com/scientificreports

[26] Singha V.K., Nagarajub Ja., Avasthi S. // Cur. Appl. Phys. 2019. V. 19. P. 341-346. doi 10.1016/j.cap.2018.12.016 